Bloß kein "Gedöns"

\title{
Intensive Beratung bringt nix beim Schleudertrauma
}

Ein Schleudertrauma ist in der Regel kein Fall für die Notaufnahme. Patienten, die sich mit entsprechenden Beschwerden in der Praxis vorstellen, können nach kurzer Information und versorgt mit ausreichend Schmerzmitteln guten Gewissens nach Hause entlassen werden.

Wie eine Studie im Fachblatt „Lancet“ gezeigt hat, tragen weder ausführliche Beratungsgespräche noch eine Physiotherapie maßgeblich zur Besserung der Beschwerden bei einem Schleudertrauma bei. Wissenschaftler von der Universität
Warwick im britischen Coventry haben 3851 Patienten nachbeobachtet, die sich nach einem Verkehrsunfall mit typischen Symptomen - Schmerzen und/oder steifer Nacken - in einer Notfallambulanz vorgestellt hatten. 2253 Patienten kamen in den Genuss einer intensiven Betreuung: Sie wurden von speziell geschulten Ärzten explizit auf die gute Prognose hingewiesen; sie wurden ermutigt, möglichst bald wieder zu normaler Aktivität zurückzukehren; sie wurden angehalten, Nackenübungen zu machen und die verordneten Analgetika auch wirklich einzunehmen; und sie wurden darüber in-

\section{Auf dem Weg in die Klinik}

\section{Junge Notärzte geizen mit Schmerzmitteln}

Bei der Erstversorgung von Unfallopfern erhält beinahe jeder zweite Schwerverletzte während des Transports zur Klinik keine ausreichende Schmerztherapie.

Zu diesem Ergebnis kamen Anästhesisten der Universitätsklinik in Lausanne: Die Auswertung von 1202 Notfallpatienten ergab, dass $43 \%$ eine unzureichende Schmerzlinderung erfahren hatten, weil sie gar kein Schmerzmittel bekommen hatten (127 Patienten) oder nicht in ausreichender Menge (391 Patienten). Gemäß dem klinikeigenen Schmerzmanagement-Protokoll soll die Schmerztherapie mit Fentanyl i.v. $(0,5-2 \mu \mathrm{g} / \mathrm{kg})$ erfolgen, bis der NRS-Wert (numerische Schmerzskala) auf 3 oder darunter sinkt. Zusätzliche Medikamente, wie Benzodiazepine oder Ketamin, bleiben der Einschätzung des Arztes überlassen.

Unterdosiert wurden die Analgetika vor allem bei schwerer verletzten Patienten, die am Unfallort eine hohe Atemfrequenz zeigten und über stärkere Schmerzen klagten. Die Ärzte orientierten sich bei der Fentanyldosierung zwar an der am Unfallort angegebenen Schmerzstärke, sie korrigierten die Menge aber nicht nach oben, wenn der NRS während des Transportes $>3$ blieb. Bei weniger starken Schmerzen (NRS <6) verzichteten die Ärzte auch gern mal ganz auf Schmerzmittel.

\section{Mit der Erfahrung steigt die Dosis}

Ärztinnen gingen sehr viel restriktiver mit Fentanyl um als die männlichen Kollegen: Oligoanalgesien waren doppelt so häufig ( $p<0,001)$. Auch die Routine schlug sich nieder: Je länger ein Arzt im Beruf arbeitete, umso effektiver bekämpfte er die Schmerzen. Unzureichend wurde am häufigsten bei Ärzten mit max. zweijähriger Berufserfahrung behandelt (OR 16,7). (dk)

Albrecht E et al, Br J Anaesth 2013, 110: 96-106 formiert, dass es sich bei den Schmerzen um eine normale Reaktion des Körpers auf das Trauma handelt. Zusätzlich wurde ein Patientenratgeber ausgehändigt, in dem ausführliche Informationen zu den vermuteten Ursachen und Folgen des Schleudertraumas nachzulesen waren.

\section{Langfristige Beschwerden bei der Mehrzahl der Patienten}

Nach zwölf Monaten zeigte sich, dass sich der Aufwand kaum gelohnt hatte: Im Neck Disability Index (NDI) schnitten die intensiv versorgten Patienten nicht besser ab als die Teilnehmer, die lediglich eine Standardinformation erhalten hatten. Mit dem NDI werden vom Patienten selbst berichtete Einschränkungen im Alltag erhoben, unter anderem beim Schlafen, Autofahren, Lesen und bei der Arbeit. Die gesamte Skala reicht von 0 (keine Einschränkung) bis 50 (maximale Behinderung). Eine spürbare Verbesserung gaben nur 42 bzw. 37\% der Patienten an. In beiden Gruppen litt also jeweils mehr als die Hälfte der Patienten noch ein Jahr nach der Kollision unter erheblichen Beschwerden; diese hatten sich teilweise sogar noch verschlimmert.

In einem zweiten Teil der Studie nahmen die Forscher um Prof. Sarah E. Lamb die Effekte einer intensiven Physiotherapie mit bis zu sechs Sitzungen unter die Lupe. Verglichen wurde mit einer einzelnen Physiotherapiesitzung. Auch hier waren die Ergebnisse langfristig bescheiden: Nach vier Monaten zeigte sich zwar ein Unterschied von 3,7 Punkten auf der NDI-Skala zugunsten der intensiv betreuten Gruppe, dieser verlor sich jedoch bereits nach acht Monaten wieder. Immerhin ging die Zahl der Arbeitsunfähigkeitstage innerhalb eines Jahres zurück (um 40\%), dafür war die Kostenbilanz aber letztlich negativ. (Elke Oberhofer)

Lamb SE et al, Emergency department treatments and physiotherapy for acute whiplash, Lancet 2012 (online first); doi: 10.1016/S0140-6736(12)61304-X 